\title{
Symbolic Analytical Developments of the Zero Pressure Cosmological Model of the Universe
}

\author{
M. A. Sharaf \\ Department of Astronomy, Faculty of Science, King Abdul Aziz University \\ Jeddah, Saudi Arabia
}

\begin{abstract}
In this paper, literal analytical solutions in power series forms are developed for the radius of curvature and the expansion velocity of the zero pressure cosmological model of the universe. For computational developments of these solutions, an efficient method using continued fraction theory is provided together with some numerical examples.
\end{abstract}

Keywords: Cosmology, cosmological models of the universe, theory of general relativity.

\section{Introduction}

Astronomical observations reveal that the universe is homogeneous and isotropic on scales of $\approx 10^{8}$ light years and larger together with the zero pressure condition. The history of this universe could be studied by computing the evolution of its scale factor. The behaviour of the scale factor, how it increases or decreases in time, describes the way of the expansion or contraction of the universe. For such studies, The differential system of the model, together with the two free parameters, the density and acceleration parameters are usually used. 
Undoubtedly, true that, the numerical integration methods can provide very accurate models. But certainly, if full analytical formulae are utilized with nowadays existing symbols used for manipulating digital computer programs, they definitely become invaluable for obtaining models with desired accuracy. Moreover, these analytical formulae usually offer much deeper insight into the nature of a model as compared to numerical integration.

In the present paper, literal analytical solutions in power series forms are developed for the radius of curvature and the expansion velocity of the zero pressure cosmological model of the universe. For computational developments of these solutions, an efficient method using continued fraction theory is provided together with some numerical examples.

\section{Einstein field equations}

\subsection{General equations}

- The three general equations from Einstein theory of general relativity that describing the structure and evolution of a spherically symmetric universe are:

$$
\begin{aligned}
& 3 \frac{\dot{R}^{2}}{R^{2}}+\frac{3 k c^{2}}{R^{2}}=8 \pi G \rho+\Lambda c^{2}, \\
& 2 \frac{\ddot{R}}{R}+\frac{\dot{R}^{2}}{R^{2}}+\frac{k c^{2}}{R^{2}}=-\frac{8 \pi G P}{c^{2}}+\Lambda c^{2}, \\
& \frac{\ddot{R}}{R}=\frac{\Lambda c^{2}}{3}-\frac{4 \pi G}{3}\left[\rho+\frac{3 P}{c^{2}}\right],
\end{aligned}
$$

where

$R(t)=$ radius of curvature of the universe, $k=$ curvature index $=0, \pm 1(k=1$,elliptical closed space ; $k=0$,Euclidean flat space; 
$k=-1$,hyperbolic open space),

$\rho=$ mean density of matter and energy,

$\Lambda=$ cosmological constant,

$P=$ hydrodynamic pressure of matter and radiation,

$G=$ gravitational constant.

- Two free parameters are usually introduced to determine the physical characteristics of a particular model of the universe:

1-The density parameter $\sigma_{0}$ :

$$
\sigma_{0}=\frac{4}{3} \pi \rho_{0} G H_{0}^{-2}
$$

2-The acceleration parameter $q_{0}$ :

$$
q_{0}=-\frac{\ddot{R}_{0}}{R_{0}} H_{0}^{-2},
$$

where, the subscript zero stands for the physical quantities at their present value and

$$
H_{0}=\frac{\dot{R}_{0}}{R_{0}}
$$

is the Hubble constant.

- These two parameters are linked with the classical parameters by the following relations : 


$$
\begin{aligned}
& \Lambda c^{2}=3 H_{0}^{2}\left(\sigma_{0}-q_{0}\right) \\
& R_{0}^{2}=k c^{2}\left(3 \sigma_{0}-q_{0}-1\right)^{-1} H_{0}^{-2} \\
& k=\operatorname{Sign}\left(3 \sigma_{0}-q_{0}-1\right)
\end{aligned}
$$

where the $\operatorname{Sign}(\mathrm{x})$ is zero, +1 or -1 for $\mathrm{x}$, zero, positive or negative.

\subsection{Physical characteristics and the classical parameters}

- The relations between the physical characteristics of a model for the universe and the classical parameters are summarized as[3]:

$\Delta$ If $\Lambda=0$

(a) $q_{0} \succ 0.5 \Leftrightarrow k \succ 0 \Leftrightarrow$ (positive curvature" closed " universe) $\Leftrightarrow$ universe will eventually recontract

(b) $q_{0}=0.5 \Leftrightarrow k=0 \Leftrightarrow$ (zero curvature" flat " universe) $\Rightarrow$ universe will expand forever

(c) $q_{0} \prec 0.5 \Leftrightarrow k \prec 0 \Leftrightarrow$ (negative curvature" open "universe) $\Rightarrow$ universe will expand forever

$\Delta$ If $\Lambda \neq 0$

(a) $\sigma_{0} \succ \frac{1}{3}\left(q_{0}+1\right) \Leftrightarrow k \succ 0$ (positive curvature" closed "universe) and in this case,

$$
\sigma_{0}-q_{0} \geq \frac{1}{\sigma_{0}^{2}}\left(\sigma_{0}-\frac{q_{0}+1}{3}\right)^{3} \Leftrightarrow \text { universe will expand forever }
$$


$\sigma_{0}-q_{0} \prec \frac{1}{\sigma_{0}^{2}}\left(\sigma_{0}-\frac{q_{0}+1}{3}\right)^{3} \Leftrightarrow$ universe will eventually recontract

(b) $\sigma_{0}=\frac{1}{3}\left(q_{0}+1\right) \Leftrightarrow k=0$ (zero curvature" flat " universe) and in this case,

$\sigma_{0} \geq q_{0} \Leftrightarrow$ universe will expand forever

$\sigma_{0} \prec q_{0} \Leftrightarrow$ universe will eventually recontract

(c) $\sigma_{0} \prec \frac{1}{3}\left(q_{0}+1\right) \Leftrightarrow k \prec 0$ (negative curvature" open " universe) and in this case,

$\sigma_{0} \geq q_{0} \Leftrightarrow$ universe will expand forever

$\sigma_{0} \prec q_{0} \Leftrightarrow$ universe will eventually recontract .

\section{Zero pressure models}

\subsection{Basic equation}

In the present paper we assume that the pressure is zero , a condition justified by the observations. The basic differential equation for these models could be obtained from the above equations as follows

- Put $P=0$ in Equation (2) then we get from Equation (1) :

$$
\ddot{R}=-\frac{4 \pi G \rho R}{3}+\frac{R \Lambda c^{2}}{3}
$$


- The conditions at which the total amount of mass has to remain constant during the evolution of the universe is

$$
\rho_{0} R_{0}^{3}=\rho(t) R(t)
$$

using this condition into the above equation we get

$$
\ddot{R}=-4 \pi G \rho_{0} \frac{R_{0}^{3}}{3 R^{2}}+\frac{\Lambda c^{2} R}{3},
$$

this is the required equation for the zero pressure cosmological model of the universe. In this equation:

$\Delta$ the first term represents the gravitational force working to keep the universe together. As usual, its value decreases with the square of the distance.

$\Delta$ The second term of the equation is the cosmological force. If $\Lambda$ is zero,gravitation is the only force. If not ,the cosmological force is directed outward if $\Lambda$ is positive and inwards in negative.

\subsection{Transformed equation}

- It is usually transform Equation (10) into a form that contains only $\sigma_{0}$ and $q_{0}$ as free parameters . This could be performed by changing both the dependent and the independent variables as follows:

$\Delta$ the dependent variable $R$ is changed to

$$
R=Y R_{0}
$$


that to say, the scale factor is taken as unit of length.

$\boldsymbol{\Delta}$ The independent variable $t$ is changed to

$$
t=X H_{0}^{-1}
$$

that is to say $H_{0}^{-1}$ is taken as unit of time, for example if we used for $H_{0}$ the value of $55 \mathrm{~km} / \mathrm{sec} / \mathrm{Mpc}$, then the unit of time is $1.801 \times 10^{10}$ years

$\boldsymbol{\Delta}$ Let " dash" denotes the differentiation with respect to $X$,so

$$
\dot{R}=H_{0} R^{\prime} \quad \text { and } \quad \ddot{R}=H_{0}^{2} R^{\prime \prime}
$$

then Equation(10) reduces by the aid of Equations (4),(7)and(11) to

$$
Y^{\prime \prime}=-\frac{\sigma_{0}}{Y^{2}}+\left(\sigma_{0}-q_{0}\right) Y
$$

This is the required transformed equation, which is free from Hubble constant and to be solved with the initial conditions:

$$
\text { at } X=0, \quad Y=1 \quad \text { and } \quad Y^{\prime}=1
$$

\subsection{Linear system}

The linear system of the zero pressure models could be obtained by letting 


$$
S=\frac{1}{Y^{2}},
$$

then Equation(13) is transformed into the linear differential system:

$$
\begin{aligned}
Y^{\prime} & =Z \\
Z^{\prime} & =-\sigma_{0} S+\left(\sigma_{0}-q_{0}\right) Y \\
S^{\prime} Y & =-2 Z S
\end{aligned}
$$

which is to be solved subject to the initial conditions :

At $X=1 \quad ; \quad Y=Z=S=1$

\section{Analytical solution}

- Undoubtedly true that, in the absence of closed analytical solution of a given differential system the power series solution (which of course assumed to be convergent) can serve as the analytical representation of its solution. Moreover, it is worth noting that the power series is one of the most powerful methods of mathematical analysis and is no less (and some - times even more) convenient than the elementary functions especially when the problems are to be studied on computers. In fact, most computers often use series in the calculations of the majority of the elementary functions .

- The power series solution of the above system[Equations(16),(17) and(18)] which satisfies the intial conditions of Equation (19) could be obtained througth the follwing steps: 
Step 1: Power series for the variables

Let

$Y=\sum_{n=1}^{\infty} y_{n} X^{n-1}$

$Z=\sum_{n=1}^{\infty} z_{n} X^{n-1}$

$S=\sum_{n=1}^{\infty} s_{n} X^{n-1}$

Step 2: Product of two infinit power series

Let $W, U$ be two infinit power series of the form:

$$
W=\sum_{i=1}^{\infty} w_{i} X^{i-1}, \quad U=\sum_{i=1}^{\infty} u_{i} X^{i-1}
$$

then

$$
F=W \times U=\sum_{n=1}^{\infty} f_{n} X^{n-1}
$$

where

$$
f_{n}=\sum_{k=1}^{n} u_{k} w_{n-k+1} ; \quad \forall n \geq 1
$$

Step 3: Substitution step 
Substituting Equations (20),(21) and (22) into Equations (16),(17) and (18) we get with the aid of Equations (23) and (24) we get

$$
\begin{aligned}
& \sum_{n=1}^{\infty} n y_{n+1} X^{n-1}=\sum_{n=1}^{\infty} z_{n} X^{n-1} \\
& \sum_{n=1}^{\infty} n z_{n+1} X^{n-1}=-\sigma_{0} \sum_{n=1}^{\infty} s_{n} X^{n-1}+\left(\sigma_{0}-q_{0}\right) \sum_{n=1}^{\infty} y_{n} X^{n-1} \\
& \sum_{n=1}^{\infty}\left[\sum_{k=1}^{n} k s_{k+1} y_{n-k+1}\right] X^{n-1}=-2 \sum_{n=1}^{\infty}\left[\sum_{k=1}^{n} z_{k} s_{n-k+1}\right] X^{n-1}
\end{aligned}
$$

Step 4: Identification of equal powers of $X$

Equating the coefficients of equal powers of $X$ in both sides of Equations (24),(26) and(27) we get the recursion equations

$$
\begin{aligned}
& n y_{n+1}=z_{n} \\
& n z_{n+1}=-\sigma_{0} s_{n}+\left(\sigma_{0}-q_{0}\right) y_{n} \\
& n s_{n+1}=-2 \sum_{k=1}^{n} z_{k} s_{n-k+1}-\sum_{k=1}^{n-1} k s_{k+1} y_{n-k+1}
\end{aligned}
$$

Step 5: Initial conditions

From Equations (19),(28),(29) and(30) we get

$$
\begin{aligned}
& y_{1}=z_{1}=s_{1}=1 \\
& y_{2}=1 ; z_{2}=-q_{0} ; s_{2}=-2
\end{aligned}
$$

These equations are applied $\forall n=2,3, \ldots, n_{t}$, where $n_{t}$ is the number of terms of the power series.If Equations (28),(29)and(30) are used in the same order as they stand, 
then all the coefficients of the power series are completely determined in a full recursive way .Finally, It should also be noted that,these series could be used for future $(X \succ 0)$ as well as for $\operatorname{past}(X \prec 0)$

Now, for given values of the parameters $\sigma_{0}, q_{0}$ and a value of $X$ one can use series (19)and(20) to compute, the radius of curvature of the universe $R(t)\left(=R_{0} Y\right)$ and its expansion velocity $\dot{R}(t)\left(=R_{0} Z H_{0}\right)$ at a any time $t\left(=X H_{0}^{-1}\right)$. In what follows we shall consider the reverse of the above problem .

\section{Inverse solution}

\subsection{Series inversion of power series}

- Genenral algorithm for reversing a power series will be developed as follows. Consider the functional equation

$$
\eta=\varsigma+\alpha \phi(\eta), \quad|\alpha| \prec 1
$$

then according to Lagrange expansion theorem [4], we have

$$
\eta=\varsigma+\sum_{n=1}^{\infty} \frac{\alpha^{n}}{n !} \frac{\mathrm{d}^{n-1}}{\mathrm{~d} \varsigma^{n-1}}[\phi(\varsigma)]^{n}
$$

- Let $h(\theta)$ be a function which can be expressed in a Taylor series in the neighborhood of $\theta=\theta_{0}$. Thus

$$
h(\theta)=h_{0}+\sum_{j=1}^{\infty} \frac{B_{j}}{j !}\left(\theta-\theta_{0}\right)^{j},
$$


where

$$
B_{j}=\left.\frac{\mathrm{d}^{j} h(\theta)}{\mathrm{d} \theta^{j}}\right|_{\theta=\theta_{0}}
$$

In the folloing,we assume that $B_{1}$ is different from zero and write Equation (35) in the form

$$
\theta=\theta_{0}+\left(h-h_{0}\right) \phi(\theta),
$$

where $\phi(\theta)$ is defined by

$$
\phi(\theta)=\frac{1}{B_{1}+\sum_{j=1}^{\infty}\left[B_{j+1} /(j+1) !\right]\left(\theta-\theta_{0}\right)^{j}} .
$$

Equation (36) is precisely the form as Equation (33), then we can express $\theta$ as a power series in $\alpha=h-h_{0}$ to get

$\theta(h)=\theta_{0}+\sum_{n=1}^{\infty} \frac{C_{n}}{n !}\left(h-h_{0}\right)^{n}$,

where

$$
C_{n}=\left.\frac{\mathrm{d}^{n-1}}{\mathrm{~d} \theta^{n-1}}[\phi(\theta)]^{n}\right|_{\theta=\theta_{0}}
$$

and $\phi(\theta)$ is defined in Equation (37). The series for $\theta(h)$ is said to be the reverse of the series for $h(\theta)$. 
Battin [1] developed an elegant algorithm to express $n$ of the coefficients $C_{1}, C_{2}, \ldots$ of the reversed series in terms of the coefficients $B_{1}, B_{2}, \ldots$ of the original series. The basic equations of this algorithm are

$$
\begin{aligned}
& \left.D_{0}^{1}\right|_{\theta=\theta_{0}}=\phi^{(0)}\left(\theta_{0}\right) \equiv \phi_{0}^{(0)} \equiv \frac{1}{B_{1}}, \\
& \frac{\mathrm{d}^{k} \phi(\theta)}{\mathrm{d} \theta^{k}} \equiv \phi_{0}^{(k)}=-\frac{1}{B_{1}} \sum_{i=1}^{k} \frac{1}{i+1}\left(\begin{array}{c}
k \\
i
\end{array}\right) B_{i+1} \phi_{0}^{(k-i)}, k=1,2, \ldots, n-1, \\
& D_{k}^{n}=\frac{\mathrm{d}^{\mathrm{k}}}{\mathrm{d} x^{k}}[\phi(\theta)]^{n}=n \sum_{j=0}^{k-1}\left(\begin{array}{c}
k-1 \\
j
\end{array}\right) D_{j}^{n-1} \phi_{0}^{(k-j)}, \\
& C_{n}=\left.D_{n-1}^{n}\right|_{x=x_{0}} .
\end{aligned}
$$

\subsection{The Inverse zero pressure model}

The inverse problem of the zero pressure cosmological model is to find the time $t=\tilde{t}$ (say) [or equivalently the value $X=\tilde{X}$ ] at which the radius of curvature of the

model $R=\widetilde{R}$ [or equivalently at the value $Y=\widetilde{Y}$ ]. The following is an algorithm to illustrate the solution of this problem

\subsubsection{Algorithm 1}

- Purpose: to find $\tilde{t}$

- Input : $\tilde{Y}, H_{0}, n_{t}$ and $y_{k} ; k=1,2, \cdots n_{t}$

\section{- Computational steps}


1-Set $B_{i+1}=(i+1) ! y_{i+2} \forall \mathrm{i}=0,1, \cdots, m\left(=n_{t}-2\right)$

2-Apply the algorithm of Subsection $\mathbf{5 . 1}$ to find the $\beta$ 's coefficients in the expansion

$$
\widetilde{X}=\sum_{k=1}^{m_{t}} \beta_{k} \frac{(\tilde{Y}-1)^{k}}{k !}
$$

3- $\tilde{t}=\tilde{X} / H_{0}$

\section{4- End}

The above algorithm could also be used to find the time at which the expansion

velocity $\widetilde{R}$ [or equivalently at the value $Z=\widetilde{Z}$ ] of the model is given. This could be done by replacing $y^{\prime}$ s by $z$ 's and $\widetilde{Y}$ by $\widetilde{Z}$ in the computational steps of algorithm 1 .

To this end, it should mentioned that ,the convergence of the fundamental power series are not studied in the present paper and will constitute a task to which we shall consider latter. However we can in general improve the convergence of a power series by means of the known Euler's device.

\section{Computational developments}

In fact, continued fraction expansions are, generally far more efficient tools for evaluating the classical functions than the more familiar infinite power series. Their convergence is typically faster and more extensive than the series. Due to the importance of accurate evaluations and the efficiency of continued fractions, I purpose to use them 
as the computational tools for evaluating zero pressure cosmological model . To do so, two steps are to be performed:

(1) Transform the given power series into continued fraction (Section 6.1).

(2) Evaluating the resulting continued fraction (Section 6.2), as follows:

\subsection{Euler's transformation}

Generally an infinite series (a power series is special case of it) of functions could be converted into a continued fraction according to Eulers transformation [1] which is

$$
\sum_{k=0}^{\infty} U_{K} \equiv \frac{n_{1}}{d_{1}+\frac{n_{2}}{d_{2}+\frac{n_{3}}{d_{3}+\frac{n_{4}}{\ldots}}}} \equiv \frac{n_{1}}{d_{1}+} \frac{n_{2}}{d_{2}+\frac{n_{3}}{d_{3}+}} \frac{n_{4}}{d_{4}+}+\ldots
$$

where

$$
\begin{aligned}
& n_{1}=U_{0} ; n_{2}=U_{1} ; n_{i}=-U_{i-1} \times U_{i-3}, \forall i \geq 3 \\
& d_{1}=1 ; d_{j}=U_{j-2}+U_{j-1} \forall i \geq 2 .
\end{aligned}
$$

\subsection{Top-down continued fraction evaluation}

There are several methods available for the evaluation of continued fraction. Traditionally, the fraction was either computed from the bottom up, or the numerator and denominator of the $n$th convergent were accumulated separately with three-term recurrence formulae. The draw back of the first method is, obviously, having to decide 
far down the fraction to being in order to ensure convergence. The draw back to the second method is that the numerator and denominator rapidly overflow numerically even though their ratio tends to a well defined limit. Thus, it is clear that an algorithm that works from top down while avoiding numerical difficulties would be ideal from a programming standpoint .

Gautschi, [2] proposed very concise algorithm to evaluate continued fraction from the top down and may be summarized as follows. If the continued fraction is written as

$$
q=\frac{n_{1}}{d_{1}+} \frac{n_{2}}{d_{2}+} \frac{n_{3}}{d_{3}+} \ldots
$$

then initialize the following parameters

$a_{1}=1, \quad b_{1}=n_{1} / d_{1}, q_{1}=n_{1} / d_{1}$

and iterate $(k=1,2, \ldots)$ according to

$$
\begin{aligned}
& a_{k+1}=\frac{1}{1+\frac{n_{k+1}}{d_{k} d_{k+1}} a_{k}} \\
& b_{k+1}=\left(a_{k}-1\right) b_{k}, \\
& q_{k+1}=q_{k}+b_{k+1} .
\end{aligned}
$$

In the limit, the q sequence converges to the value of the continued fraction

\subsection{Numerical examples}

In the following numerical applications we consider the value of the Hubble constant as $H_{0}=55 \mathrm{~km} / \mathrm{sec} / \mathrm{Mpc}$ 
- $\quad$ For $\sigma_{0}=q_{0}$

1- $\sigma_{0}=q_{0}=0.4, X=0.02\left(t=35.02 \times 10^{7}\right.$ years $) \Rightarrow Y=1.01992105$

2- $\sigma_{0}=q_{0}=0.6, X=-0.04\left(t=-72.04 \times 10^{7}\right.$ years $) \Rightarrow Y=0.9595067$

3- $\sigma_{0}=q_{0}=0.5, X=0.4\left(t=720.4 \times 10^{7}\right.$ years $) \Rightarrow Y=1.36797989$

- $\quad$ For $\sigma_{0} \neq q_{0}$

4- $\sigma_{0}=0.5, q_{0}=0.34, X=0.03\left(t=54.03 \times 10^{7}\right.$ years $) \Rightarrow Y=1.02985211$

5- $\sigma_{0}=1.0, q_{0}=2.0, X=-0.1\left(t=-180.1 \times 10^{7}\right.$ years $) \Rightarrow Y=0.88979408$

6- $\sigma_{0}=4.0, q_{0}=5.0, X=0.1\left(t=180.1 \times 10^{7}\right.$ years $) \Rightarrow Y=1.07595569$

In concluding the present paper, literal analytical solutions in power series forms are developed for the radius of curvature and the expansion velocity of the zero pressure cosmological model of the universe.The importance of these solutions are due their analytical forms, which offer in general much deeper insight into the nature of the physical characteristics to which they refer.

On the other hand we proposed for the computational developments of these expressions the continued fraction theory. The continued fraction not given the prominence they deserve in the university curricula despite the fact that they are, generally, far more efficient tools for evaluating the classical functions than the more familiar infinite power series. Their convergence is typically faster and more extensive than the series and, ironically, they were in use centuries before the invention of the power series.Finally some numerical examples are also given.

\section{References}

1- Battin, R. H.:1999, An Introduction to the Mathematics and Methods of Astrodynamics, Revised Edition, AIAA, Education Series, Reston, Virginia.

2- Gautschi, W. : 1967, Computational Aspects of Three-term Recurrence Relations, SIAM Review, Vol. 9, No. 1, January. 
3-Misner,C.,Thorne,K. and Wheeler,A.:1973,Gravitation, Freeman and Company,New York

4-Smart,W.M.:1953,Celestial Mechanics,Longmans,Green and Co.,London

Received: August, 2005 